\title{
Bridge-Ship Collision Avoidance Control Based on AFSMC with a FRNN Estimator
}

\author{
Qionglin Fang $\mathbb{D}^{1}$ and Enguang $\mathrm{Cao}^{2}$ \\ ${ }^{1}$ College of Navigation, Jimei University, Xiamen 361021, Fujian, China \\ ${ }^{2}$ Lianyungang Pilot Station, Lianyungang 222000, Jiangsu, China
}

Correspondence should be addressed to Qionglin Fang; fq11437@163.com

Received 23 April 2021; Revised 5 July 2021; Accepted 14 July 2021; Published 22 July 2021

Academic Editor: Rahib Abiyev

Copyright (C) 2021 Qionglin Fang and Enguang Cao. This is an open access article distributed under the Creative Commons Attribution License, which permits unrestricted use, distribution, and reproduction in any medium, provided the original work is properly cited.

\begin{abstract}
For collision avoidance and maneuvering control in bridge areas, an adaptive fractional sliding mode control with fractional recurrent neural network (FRNN-AFSMC) is proposed. The uncertainties are estimated by FRNN, and the fractional gradient is adopted to improve the recurrent neural network (RNN). Its convergence has been proven. The influence of fractional order on algorithm performance is analyzed, and the simulation platform of ship collision avoidance control is built. Dynamic collision avoidance of multiple ships is simulated and verified. The results show the feasibility and effectiveness of dynamic autonomous collision avoidance motion control in a dynamic ocean environment.
\end{abstract}

\section{Introduction}

Collision avoidance and maneuvering control in bridge areas are important for the autonomous intelligent navigation in ships. Due to the large inertia, nonlinearity and uncertainty of ship motion, underactuated characteristics, and strong external interferences such as wind, waves, and current, automatic collision avoidance navigation and motion control in ships is a great challenge. Wu proposed a fuzzy logic approach for ship-bridge collision alerts [1]. Li discussed the unsteady hydrodynamic loads of ships passing near bridge piers [2]. Sha discussed bridge girders against ship forecastle collision loads [3]. Pedersen studied the design of bridges against ship collisions [4]. Fang studied composite bumper systems for bridge pier protection against ship collisions [5]. Zhu discussed lattice composite bumper systems for bridge protection in ship collisions [6].

Although some ships are equipped with side thrusters, they do not work at constant speed. How to design robust controllers for underactuated ships has become a key problem. Variable structure control has become an effective control method for ships. To improve the control effect of sliding mode, fractional calculus is introduced. It helps to obtain faster response speeds, lower overshoot, smaller chattering effects, and better control performance. Fei introduced a fractional-order sliding mode control method based on recursive neural network approximation [7]. To improve the output power quality of a permanent magnet synchronous generator, Xiong proposed a fractional-order sliding mode control method [8]. Sharafian proposed a new fractional-order observer based on a sliding mode method and radial basis function neural network to distinguish the uncertainty of a fractional-order human immunodeficiency virus mathematical dynamic model [9]. Yang proposed novel passive fractional-order sliding mode control for distributed generator microgrid supercapacitor energy storage systems [10]. Moezi proposed a new adaptive interval type 2 fuzzy fractional backstepping sliding mode control method [11]. Ren proposed a fractional-order sliding mode controller based on an exponential reaching law to control the pneumatic position servo system [12]. To approximate the system uncertainty at sea, a neural network is used. A recurrent neural network $(\mathrm{RNN})$ is a kind of neural network that inputs sequence data, and all its nodes are connected by chain. In 1990, the first fully connected RNN, namely, the Elman network, was proposed 
[13]. A new observer structure for nonlinear fractionalorder systems was proposed by Sharafian to estimate the states of fractional-order nonlinear chaotic systems with unknown dynamic models [14]. A new fractional-order error back-propagation learning algorithm was proposed to adapt to the weights of neural networks by using the Lyapunov stability strategy of fractional-order system, called Mittag-Leffler stability. The main contribution was to extend the neural observer of fractional-order dynamics by satisfying the Mittag-Leffler condition. The observer design process ensured that the observer error converged to the zero point. For a class of nonlinear fractional multiagent systems, five special types of sliding surfaces were proposed by Sharafian to overcome the consistency tracking problem [15]. The disturbance suppression control input could stabilize the uniform error dynamics of fractional-order multiagent system. Integer-order and fractional-order sliding surfaces were used to improve the accuracy and convergence speed. Based on Lyapunov direct strategy and Mittag-Leffler stability of fractional derivative, the stability of various sliding surfaces was proved. In order to show that the sliding motion was generated in finite time, the upper bound of the arrival time of the sliding surface was given. Based on the dynamic memory reset principle of fractional-order operators, it was forced to be an invariant finite time sliding mode. Jesus considered an optimal fuzzy fractional PD + I controller in which the parameters were tuned by a GA [16]. The performance of the proposed fuzzy fractional control was illustrated through some application examples. Abiyev introduced the development of fractional-order controller for dynamic control [17]. The parameters of fractional-order controller were adjusted by real coded genetic algorithm.

To update and optimize the weights of neural networks, gradient descent (GD) is a basic method. The main contributions of this paper are as follows:

(1) Fractional calculus is adopted to improve RNN for approximating unknown parameters of ship model and environmental disturbance

(2) FRNN-AFSMC is designed, and its convergence is proven

(3) The proposed algorithm is used for collision avoidance of underactuated ships through course alterations

\section{Preliminaries and Problem Statement}

2.1. Ship Drift in Bridge Areas. Assume that the origin of the calculation coordinate is the center of the channel. The longitudinal axis $X$ is perpendicular to the bridge axis and points downstream. The transverse axis $Y$ is parallel to the bridge axis and points to the left bank. When a ship crosses a bridge, the yaw angle is $\alpha$, and the flow direction angle is $\beta$. Then the drift $B_{1}$ of the ship under the influence of currents can be calculated as follows:

$$
B_{1}=S \frac{V \sin \alpha+U \sin \beta}{|V \cos \alpha+U \cos \beta|^{\prime}}
$$

where $S$ is the length of the bridge, $V$ is the speed of the ship, and $U$ is the speed of the current.

The drift $\mathrm{B} 2$ by wind is

$$
B_{2}=K \sqrt{\frac{B_{a}}{B_{w}}} e^{-0.1 W_{s}} V_{a} S \frac{\sin \alpha_{f}}{|V \cos \alpha+U \cos \beta|},
$$

where $K$ is the coefficient, $B_{\mathrm{a}}$ is the wind area above the hull waterline, $B_{\mathrm{W}}$ is the area below the hull waterline, $V_{a}$ is the relative wind speed, and $\alpha_{\mathrm{f}}$ is the angle between the wind direction and the normal of the bridge axis.

2.2. Ship Collision Avoidance. When the ship is sailing in narrow waters and there is a risk of collision, the ship chooses course alterations to avoid collision. The optimal ship course alteration is based on the collision risk and limited feasible solution space. In the process of collision avoidance, after one or two ships take collision avoidance measures, the nearest distance DCPA (Distance of Closest Point of Approach) and the nearest time TCPA (Time to Closest Point of Approach) of the two ships must change.

For example, the true course (TC) of a ship is $011^{\circ}$ and its speed is $10 \mathrm{kn}$. The data of incoming ships observed by radar are listed in Table 1.

A basic calculation shows that the DCPA is $1.2 n$ mile. Assume that the target ship maintains this direction and speed. The baseline ship wants to maintain its speed and turn right at $09: 15$, so that the target ship passes $2.0 n$ mile away. The turning range and time to restore the original course are to be calculated. The calculation steps are as follows:

(1) Make a triangle of relative motion.

(2) Determine the relative position A4 of the target ship at $09: 15$.

(3) Create a tangent of distance circle through A4 with $\mathrm{DCPA}=2 n$ mile and obtain A4Y1.

(4) The parallel line A3Y11 of A4Y1 is drawn through point A3.

(5) Take point $M$ as the center of the circle and MA1 as the radius and draw an arc. It crosses A3Y11 at point A11. MA11 is the motion vector of the ship's turning avoidance. The bearing difference between MA11 and MA1 is the turning angle.

(6) Make a parallel line of the A1A3 tangent to the $2.0 n$ mile distance circle and intersect $\mathrm{Y} 1$ at point A5. When the target ship arrives at $\mathrm{A} 5$, the original course of the ship is restored. The relative motion vector of the target ship is restored to A1A3. The actual relative motion line is $\mathrm{A} 5 \mathrm{Y} 2$, and it passes 2.0 $n$ mile away. This process is shown in Figure 1.

2.3. Recurrent Neural Network. Figure 2 shows its architecture diagram.

Recurrent neural network takes sequence data as input and recurses in the evolution direction of sequence, and all nodes (cyclic units) are connected by chain. The output of a sequence is also related to the previous output. The specific 
TABLE 1: Data of incoming ships observed by radar.

\begin{tabular}{lccc}
\hline Time & True course $\left({ }^{\circ}\right)$ & Bearing $\left(^{\circ}\right)$ & Distance $(n$ mile $)$ \\
\hline $09: 00$ & 052 & 041 & 10.0 \\
$09: 06$ & 051 & 040 & 8.5 \\
$09: 12$ & 048 & 038 & 7.0 \\
\hline
\end{tabular}

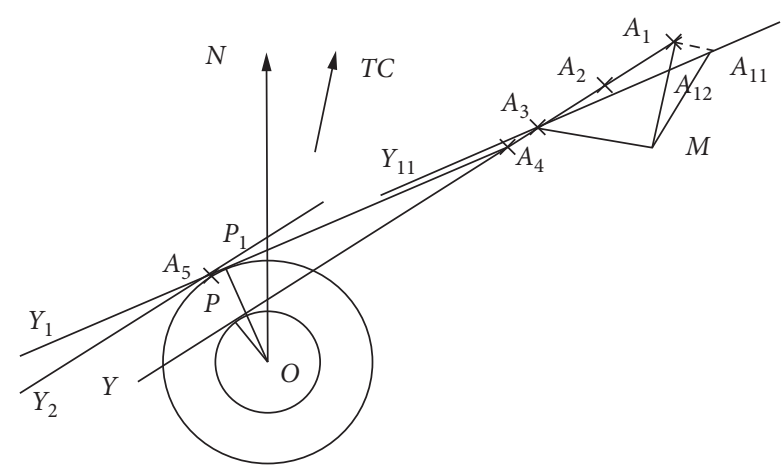

FIGURE 1: Ship collision avoidance.
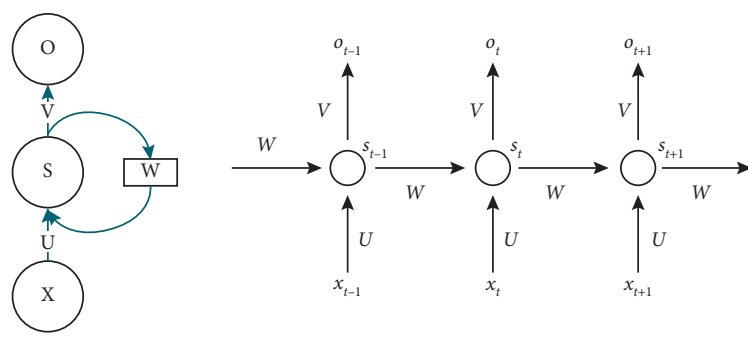

Figure 2: The architecture diagram of RNN.

form is that the network will remember the previous information and apply it to the calculation of the current output. The input of the hidden layer includes not only the output of the input layer but also the output of the previous hidden layer. Each arrow represents a transformation, and the arrow connection has weight. In the expanded structure, in the standard RNN structure, the neurons in the hidden layer also have weights. With the continuous progress of the sequence, the former hidden layer will affect the later hidden layer. $O$ represents the output, $Y$ represents the determined value given by the sample, and $L$ represents the loss function. The loss function is also accumulated with the recommendation of the sequence. Each input value of RNN only establishes a weighted connection with its own route.

The error signal is defined as [18]

$$
e(t)=o(t)-y(t) .
$$

The loss function is defined as

$$
L(t)=\frac{1}{2} \sum_{k=1}^{m} e_{k}^{2}(t)
$$

where

$$
\begin{gathered}
\Delta w(t)=w(t+1)-w(t), \\
v_{k}(t)=\sum_{j=1}^{m} w_{k j}(t) y(t) .
\end{gathered}
$$

\subsection{Fractional-Order Calculus}

Definition 1. For the absolute integrable function $x(t)$ in the interval $\left[t_{0}, t\right]$, its Riemann-Liouville integral is as follows:

$$
{ }_{t_{0}} D_{t}^{\alpha} x(t)=\frac{1}{\Gamma(\alpha)} \int_{t_{0}}^{t}(t-\tau)^{\alpha-1} x(\tau) \mathrm{d} \tau,
$$

where $\alpha$ is the fractional order, whose real part is positive. $\Gamma(x)$ is the gamma function defined as

$$
\Gamma(x)=\int_{0}^{\infty} e^{-t} t^{x-1} \mathrm{~d} t .
$$

Definition 2. For the absolute integrable function $x(t)$ in the interval $\left[t_{0}, t\right]$, its Riemann-Liouville differential is defined as

$$
t_{0} D_{t}^{\alpha} x(t)=\frac{\mathrm{d}^{m}}{\mathrm{~d} t^{n}}\left[\frac{1}{\Gamma(m-\alpha)} \int_{t_{0}}^{t}(t-\tau)^{m-\alpha-1} x(\tau) \mathrm{d} \tau\right],
$$

where $\alpha \in[m-1, m), m$ is a positive integer.

Definition 3. For the function $f(x)=\left(x-x_{0}\right)^{v}$, the following formula holds:

$$
{ }_{x_{0}} D_{x}^{p} f(x)=\frac{\Gamma(v+1)}{\Gamma(v-p+1)}\left(x-x_{0}\right)^{v-p} .
$$

2.5. Motion Model of an Underactuated Ship. The motion model is shown in Figure 1. $\varphi=\left[\varphi_{1}, \varphi_{2}, \ldots, \varphi_{P}\right]$ denotes the position and attitude, $x$ denotes the velocity, $\|x\|$ denotes the surge velocity, $x$ denotes the sway velocity, $\varphi_{i}$ denotes the yaw velocity, $x_{i}$ denotes the surge position, $\sigma_{j}$ denotes the sway position, and $\mu_{i}$ denotes the yaw angle. Figure 3 shows the ship motion model.

The mathematical model is as follows:

$$
\begin{aligned}
& \dot{\eta}=\mathbf{R}(\psi) v, \\
& \eta=\left[\begin{array}{lll}
x & y & \psi
\end{array}\right]^{T}, \\
& v=\left[\begin{array}{lll}
u & v & r
\end{array}\right]^{T},
\end{aligned}
$$

where $R$ is the rotation matrix and it can be calculated as

$$
\mathbf{R}=\left[\begin{array}{ccc}
\cos \psi & -\sin \psi & 0 \\
\sin \psi & \cos \psi & 0 \\
0 & 0 & 1
\end{array}\right]
$$

The dynamic models of underactuated ships can be calculated as follows: 


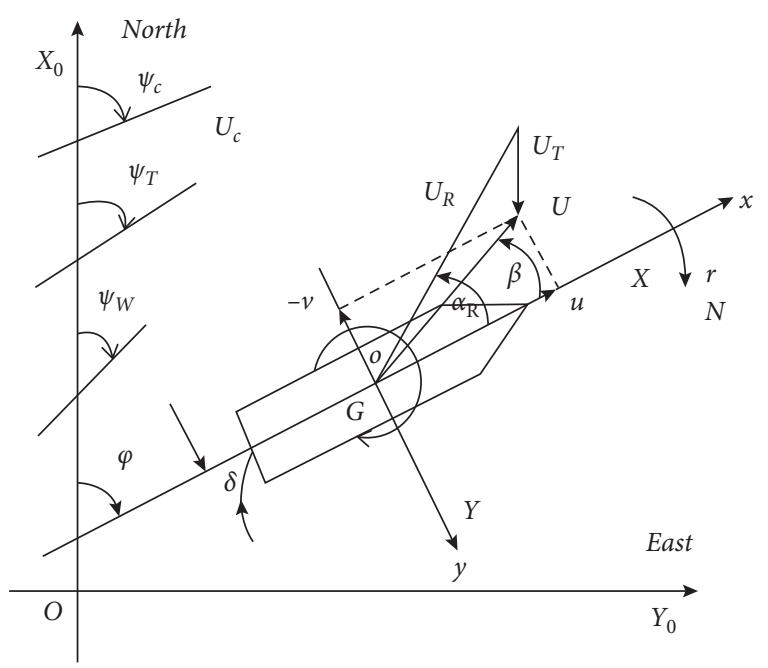

FIgURE 3: Ship motion.

$$
\left\{\begin{array}{l}
\dot{x}=u \cos \psi-v \sin \psi \\
\dot{y}=u \sin \psi+v \cos \psi \\
\dot{\psi}=r
\end{array}\right.
$$

The velocity equation is as follows:

$$
\left\{\begin{array}{l}
\dot{u}=\frac{m_{22}}{m_{11}} v r-\frac{d_{11}}{m_{11}} u+\frac{1}{m_{11}}\left(\tau_{u}+F_{w x}+F_{c x}+F_{s x}+F_{x b}\right), \\
\dot{v}=-\frac{m_{11}}{m_{22}} u r-\frac{d_{22}}{m_{22}} v+\frac{1}{m_{122}}\left(F_{w y}+F_{c y}+F_{s y}\right) \\
\dot{r}=\frac{m_{11}-m_{22}}{m_{33}} u v-\frac{d_{33}}{m_{33}} r+\frac{\tau_{r}+N_{w c}+N_{c}+N_{s}+N_{b}}{m_{33}}
\end{array}\right.
$$

where $F_{w x}, F_{w y}$, and $N_{w c}$ are the wind disturbance, $F_{c x}, F_{c y}$, and $N_{c}$ are the force of current disturbance, $F_{s x}, F_{s y}$, and $N_{s}$ are the force of wave disturbance, and $\tau_{u}$ and $\tau_{r}$ denote the control input. $\left[\begin{array}{ccc}m_{u} & 0 & 0 \\ 0 & m_{v} & m_{23} \\ 0 & m_{32} & m_{r}\end{array}\right]$ represents the weight inertia and hydrodynamic additional inertia. $\left[\begin{array}{ccc}d_{11} & 0 & 0 \\ 0 & d_{22} & d_{23} \\ 0 & d_{32} & d_{33}\end{array}\right]$ is the linear hydrodynamic damping parameter matrix. Denote

$$
\begin{aligned}
& f_{u}=\frac{m_{22}}{m_{11}} v r-\frac{d_{11}}{m_{11}} u+\frac{1}{m_{11}}\left(F_{w x}+F_{c x}+F_{s x}\right), \\
& f_{v}=-\frac{m_{11}}{m_{22}} u r-\frac{d_{22}}{m_{22}} v+\frac{1}{m_{122}}\left(F_{w y}+F_{c y}+F_{s y}\right), \\
& f_{r}=\frac{m_{11}-m_{22}}{m_{33}} u v-\frac{d_{33}}{m_{33}} r+\frac{1}{m_{33}}\left(N_{w c}+N_{c}+N_{s}\right),
\end{aligned}
$$

and (8) can be written as

$$
\left\{\begin{array}{l}
\dot{u}=f_{u}+\frac{1}{m_{11}} \tau_{u}, \\
\dot{v}=f_{v}, \\
\dot{r}=f_{r}+\frac{1}{m_{33}} \tau_{r} .
\end{array}\right.
$$

\subsection{Environmental Disturbing Force}

2.6.1. Wind Disturbance Model. The wind disturbance is

$$
\left\{\begin{array}{l}
F_{w x}=\frac{1}{2} C_{X}\left(\gamma_{r}\right) \rho_{a} A_{f} V_{r}^{2}, \\
F_{w y}=\frac{1}{2} C_{Y}\left(\gamma_{r}\right) \rho_{a} A_{s} V_{r}^{2}, \\
N_{w c}=\frac{1}{2} C_{N}\left(\gamma_{r}\right) \rho_{a} A_{s} L_{o a} V_{r}^{2},
\end{array}\right.
$$

where $C_{X}, C_{Y}$, and $C_{N}$ denote the wind coefficients. $A_{f}$ denotes the projection area above the water line. $A_{s}$ denotes the projection area on the side. $L_{o a}$ denotes the total length of the ship. $\rho_{a}$ denotes the air density.

2.6.2. Current Disturbance Model. The ocean current disturbance is

$$
\left\{\begin{array}{l}
F_{c x}=\frac{1}{2} \rho A_{f w} V_{C}^{2} C_{x}(\beta), \\
F_{c y}=\frac{1}{2} \rho A_{s w} V_{C}^{2} C_{y}(\beta), \\
N_{c}=\frac{1}{2} \rho A_{f w} L V_{C}^{2} C_{n}(\beta),
\end{array}\right.
$$

where $F_{c x}, F_{c y}$, and $N_{c}$ are the current coefficients. $V_{c}$ represents the velocity of the current. $A_{f w}$ denotes the orthographic projection area of the ship underwater. $A_{s w}$ denotes the side projection area of the ship underwater. $L$ denotes the length of the ship's waterline. $\beta$ denotes the drift angle. $\rho$ denotes the density of the sea water. $C_{x}, C_{y}, C_{n}$ denote the flow force coefficients.

2.6.3. Wave Disturbance Model. The wave disturbance is

$$
\left\{\begin{array}{l}
F_{s x}=\frac{1}{2} \rho L a^{2} \cos \chi C_{x w}(\lambda), \\
F_{s y}=\frac{1}{2} \rho L a^{2} \sin \chi C_{y w}(\lambda), \\
N_{s}=\frac{1}{2} \rho L^{2} a^{2} \sin \chi C_{n w}(\lambda),
\end{array}\right.
$$


where $a$ denotes the average wave amplitude. $\chi$ denotes the encounter angle. $C_{x w}, C_{y w}$, and $C_{n w}$ denote wave coefficients. $\lambda$ is the length of the wave.

\section{Control Design}

3.1. FRNN Algorithm. The theoretical basis of gradient descent method is the concept of gradient. The relationship between gradient and directional derivative is as follows: the direction of gradient is consistent with the direction of obtaining the maximum value of directional derivative, and the modulus of gradient is the maximum value of directional derivative of function at this point. The weight is updated using the gradient method:

$$
w_{k j}(t+1)=w_{k j}(t)-\eta D_{w_{k j}}^{\alpha} L(t),
$$

where $w_{k j}$ is the weight, $L$ is the loss function of the neural network, $\eta$ is the learning rate coefficient, and $a$ is the fractional order.

The fractional order derivative of $L(t)$ is

$$
D_{w_{k j}}^{\alpha} L(t)=\frac{\partial L(t)}{\partial v_{k}(t)} \frac{w_{k j}^{1-\alpha}(t) y_{j}(t)}{\Gamma(2-\alpha)},
$$

where $v$ is the weight from the hidden layer to the output layer of the network.

The derivative of $L(t)$ is

$$
\nabla_{w_{k j}} L(t)=\frac{\partial L(t)}{\partial v_{k}(t)} y .
$$

Substituting (25) into the first item of (24) yields

$$
w_{k j}(t+1)=w_{k j}(t)-\eta \frac{\partial L(t)}{\partial v_{k}(t)} \frac{w_{k j}^{1-\alpha}(t) y_{j}(t)}{\Gamma(2-\alpha)},
$$

where $y$ is the output of the training samples.

Substituting (27) into the first item of (5) yields

$$
\Delta w_{k j}(t)=-\eta \frac{\partial L(t)}{\partial v_{k}(t)} \frac{w_{k j}^{1-\alpha}(t) y_{j}(t)}{\Gamma(2-\alpha)} .
$$

Substituting (28) into the first item of (26) yields

$$
\nabla_{w_{k j}} L(t)=-\frac{\Gamma(2-\alpha)}{\eta} w_{k j}^{\alpha-1}(t) \Delta w_{k j}(t) .
$$

\subsection{Convergence Analysis of FRNN}

Lemma 1. The loss function $L$ is monotonically decreasing:

$$
L(w(t+1)) \leq L(w(t)) .
$$

Proof. From the Taylor mean value theorem with Lagrange remainder, we have

$$
\Delta L=L(w(t+1))-L(w(t))=L^{\prime} \Delta w(t)+\frac{1}{2} L^{\prime \prime}\|\Delta w(t)\|^{2} .
$$

Applying (29) to (31), we have

$$
\Delta L<\left[-\frac{\Gamma(2-\alpha)}{\eta} w^{\alpha-1}(t)+c\right]\|\Delta w(t)\|^{2},
$$

where $c>1 / 2 L^{\prime \prime}$. From (32), one can obtain that (30) will hold if the following is satisfied:

$$
\eta \leq \frac{\Gamma(2-\alpha)\|w(t) \mid\|^{\alpha-1}}{c} .
$$

The proof of Lemma 1 is completed.

Lemma 2. The set $\{L(t)\}$ is convergent:

$$
\lim _{t \longrightarrow \infty} L(w(t))=L^{*} .
$$

Proof. From (4), we have $L(w(t)) \geq 0$.

Every bounded monotonic sequence of real numbers converges. From Lemma $1, L(w(t+1)) \leq L(w(t))$. Therefore, there exists $L^{*} \geq 0$ satisfying

$$
\lim _{t \longrightarrow \infty} L(w(t))=L^{*} \text {. }
$$

The proof of Lemma 2 is completed.

Theorem 1. Assume that (33) is valid. Then, the set $\left\{D_{w(t)}^{\alpha} L(w(t))\right\}$ converges to zero.

$$
\lim _{t \longrightarrow+\infty}\left\|D_{w(t)}^{\alpha} L(w(t))\right\|=0
$$

Proof. Denote

$$
\lambda=\frac{\Gamma(2-\alpha)}{\eta} w^{\alpha-1}(t)-c \geq 0
$$

From (32), one can obtain

$$
\begin{aligned}
& L(w(t+1)) \\
& \quad \leq L(w(t))-\left[\frac{\Gamma(2-\alpha)}{\eta} w^{\alpha-1}(t)-c\right]\|\Delta w(t)\|^{2} \\
& \quad=L(w(t))-\lambda\|\Delta w(t)\|^{2} \\
& \quad=L(w(0))-\lambda \sum_{t=0}^{k}\|\Delta w(t)\|^{2} .
\end{aligned}
$$

Since $L(w(t)) \geq 0$, we have

$$
\lambda \sum_{t=0}^{k}\|\Delta w(t)\|^{2} \leq L(w(0)) .
$$

When $t \longrightarrow+\infty$, it holds that

$$
\sum_{t=0}^{\infty}\|\Delta w(t)\|^{2} \leq \infty .
$$

Thus, we have 


$$
\lim _{t \rightarrow \infty}\|\Delta w(t)\|=0 .
$$

Substituting (24) into (5) yields

$$
\Delta w(t)=-\eta D_{w(t)}^{\alpha} L(t)
$$

Substituting (42) into (41) yields

$$
\lim _{t \longrightarrow \infty}\left\|-\eta D_{w(t)}^{\alpha} L(t)\right\|=0 .
$$

Thus, we have

$$
\lim _{t \longrightarrow+\infty}\left\|D_{w(t)}^{\alpha} L(w(t))\right\|=0 .
$$

From Theorem 1, we conclude that FRNN converges.

3.3. Neural Fractional-Order Sliding Mode Control. Denote $\eta_{d}$ as the desired position and attitude. Denote $v_{d}$ as the desired velocity.

$$
\begin{gathered}
\eta_{d}=\left[\begin{array}{l}
x_{d} \\
y_{d} \\
\psi_{d}
\end{array}\right], \\
v_{d}=\left[\begin{array}{l}
u_{d} \\
v_{d} \\
r_{d}
\end{array}\right] .
\end{gathered}
$$

The tracking error of position and attitude is

$$
\left\{\begin{array}{l}
\bar{x}=x-x_{d}, \\
\bar{y}=y-y_{d}, \\
\bar{\psi}=\psi-\psi_{d} .
\end{array}\right.
$$

The tracking error of velocity is

$$
\left\{\begin{array}{l}
\bar{u}=u-u_{d}, \\
\bar{v}=v-v_{d}, \\
\bar{r}=r-r_{d} .
\end{array}\right.
$$

The following fractional sliding surface functions are constructed:

$$
s_{1}(t)=\bar{u}+c_{1} D^{-\alpha} \bar{u},
$$

where $c_{1}>0 . \alpha$ is the fractional order.

The derivative of (49) is obtained:

$$
\dot{s}_{1}(t)=\dot{u}-\dot{u}_{d}+c_{1} D^{1-\alpha} \bar{u}
$$

Substituting (20) into (50), one can obtain

$$
\dot{s}_{1}(t)=f_{u}+\frac{1}{m_{11}} \tau_{u}-\dot{u}_{d}+c_{1} D^{1-\alpha} \bar{u} .
$$

The sliding mode control law is constructed:

$$
\tau_{u}=m_{11}\left(\dot{u}_{d}-c_{1} D^{1-\alpha} \bar{u}-\eta_{1} \operatorname{sgn} s_{1}-\widehat{f}_{u}\right),
$$

where $\eta_{1}>0 . \widehat{f}_{u}$ is the approximation of $f_{u}$ :

$$
\begin{aligned}
\widehat{f}_{u} & =\widehat{W}_{u}^{T} \phi, \\
f_{u} & =W_{u}^{T} \phi+\varepsilon_{1},
\end{aligned}
$$

where $\varepsilon_{1}$ is the approximation error.

Subtracting (53) from (54),

$$
f_{u}-\widehat{f}_{u}=W_{u}^{T} \phi+\varepsilon_{1}-\widehat{W}_{u}^{T} h=\left(W_{u}^{T}-\widehat{W}_{u}^{T}\right) \phi+\varepsilon_{1} \text {. }
$$

Denote

$$
\widetilde{W}_{u}=W_{u}-\widehat{W}_{u} .
$$

(55) can be written as

$$
f_{u}-\widehat{f}_{u}=\widetilde{W}_{u} \phi+\varepsilon_{1} .
$$

The adaptive control law is constructed:

$$
\dot{\widehat{W}}_{u}=\frac{1}{\lambda_{1}} s_{1} \phi
$$

The fractional sliding surface is constructed:

$$
s_{2}(t)=c_{2} \bar{v}+D^{\beta} \bar{v}
$$

where $c_{2}>0 . \beta$ is the fractional order.

The derivative of (59) is obtained:

$$
\dot{s}_{2}(t)=c_{2} \dot{\bar{v}}+D^{1+\beta} \bar{v}=c_{2} \dot{\bar{v}}+D^{\beta-1} \ddot{\bar{v}}
$$

Substituting (48) into (60), one can obtain

$$
\dot{s}_{2}(t)=c_{2} \dot{\bar{v}}+D^{\beta-1}\left(\ddot{v}-\ddot{v}_{d}\right) \text {, }
$$

and $v_{d}$ is constructed as follows:

$$
v_{d}=-\sin \psi \dot{x}_{d}+\cos \psi \dot{y}_{d}+\eta_{2} \bar{x} \sin \psi-\eta_{2} \bar{y} \cos \psi .
$$

The derivative of (62) is obtained:

$$
\begin{aligned}
\dot{v}_{d}= & -\sin \psi \ddot{x}_{d}-\cos \psi r \dot{x}_{d}+\cos \psi \ddot{y}_{d}-\sin \psi r \dot{y}_{d} \\
& +\eta_{2} \dot{\bar{x}} \sin \psi+\eta_{2} \cos \psi r \bar{x}-\eta_{2} \dot{\bar{y}} \cos \psi+\eta_{2} \bar{y} \sin \psi r
\end{aligned}
$$

and $u_{d}$ is constructed as follows:

$$
u_{d}=\cos \psi \dot{x}_{d}+\sin \psi \dot{y}_{d}-\eta_{2} \bar{x} \cos \psi-\eta_{2} \bar{y} \sin \psi .
$$

The derivative of (64) is obtained:

$$
\begin{aligned}
\dot{u}_{d}= & \cos \psi \ddot{x}_{d}-\sin \psi r \dot{x}_{d}+\sin \psi \ddot{y}_{d}+\cos \psi r \dot{y}_{d} \\
& -\eta_{2} \dot{\bar{x}} \cos \psi+\eta_{2} \sin \psi r \bar{x}-\eta_{2} \dot{\bar{y}} \sin \psi-\eta_{2} \bar{y} \cos \psi r .
\end{aligned}
$$

It can be obtained by calculation that 


$$
\begin{aligned}
-2 r \dot{u}_{d}+r^{2} v_{d}= & -2 r\left[\cos \psi \ddot{x}_{d}-\sin \psi r \dot{x}_{d}+\sin \psi \ddot{y}_{d}+\cos \psi r \dot{y}_{d}-\eta_{2} \dot{\bar{x}} \cos \psi+\eta_{2} \sin \psi r \bar{x}-\eta_{2} \dot{\bar{y}} \sin \psi-\eta_{2} \bar{y} \cos \psi r\right] \\
& +r^{2}\left[-\sin \psi \dot{x}_{d}+\cos \psi \dot{y}_{d}+\eta_{2} \bar{x} \sin \psi-\eta_{2} \bar{y} \cos \psi\right] \\
= & r^{2} \sin \psi \dot{x}_{d}-r^{2} \cos \psi \dot{y}_{d}-\eta_{2} r^{2} \sin \psi \bar{x}+\eta_{2} r^{2} \cos \psi \bar{y}-2 r \cos \psi \ddot{x}_{d}-2 r \sin \psi \ddot{y}_{d} \\
& +2 \eta_{2} r \cos \psi \dot{\bar{x}}+2 \eta_{2} r \sin \psi \dot{\bar{y}} .
\end{aligned}
$$

The derivative of (63) can obtain

$$
\begin{aligned}
\ddot{v}_{d}= & -\sin \psi \ddot{x}_{d}-\cos \psi r \ddot{x}_{d}-\cos \psi r \ddot{x}_{d}+\sin \psi r^{2} \dot{x}_{d}-\cos \psi \dot{r} \dot{x}_{d}+\cos \psi \ddot{y}_{d}- \\
& \cdot \sin \psi r \ddot{y}_{d}-\sin \psi r \ddot{y}_{d}-\sin \psi \dot{r} \dot{y}_{d}+\eta_{2} \ddot{\bar{x}} \sin \psi+\eta_{2} \dot{\bar{x}} \cos \psi r+\eta_{2} \cos \psi r \dot{\bar{x}} \\
& +\eta_{2} \cos \psi \dot{r} \bar{x}-\eta_{2} \sin \psi r^{2} \bar{x}-\eta_{2} \ddot{\bar{y}} \cos \psi+\eta_{2} \dot{\bar{y}} r \sin \psi+\eta_{2} \dot{\bar{y}} \sin \psi r+\eta_{2} \bar{y} \cos \psi r^{2}+\eta_{2} \bar{y} \dot{r} \sin \psi .
\end{aligned}
$$

Substituting (66) into (67), one can obtain

$$
\ddot{v}_{d}=\ddot{y}_{d} \cos \psi-\sin \psi \ddot{x}_{d}+\eta_{2} \ddot{\bar{x}} \sin \psi-\eta_{2} \ddot{\bar{y}} \cos \psi+r^{2} v_{d}-2 r \dot{u}_{d}-\dot{r} u_{d} .
$$

The control rate is designed as follows:

$$
\tau_{r}=\frac{m_{33}}{u_{d}}\left[-\ddot{v}-D^{1-\beta}\left(k_{2} \operatorname{sgn} s_{2}+\eta_{2} s_{2}+c_{2} \dot{\bar{v}}\right)+\stackrel{\dot{t}}{y}_{d} \cos \psi-\sin \psi \stackrel{\dot{\mathrm{t}}}{x_{d}}+\eta_{2} \ddot{\bar{x}} \sin \psi-\eta_{2} \ddot{\bar{y}} \cos \psi-2 r \dot{u}_{d}+r^{2} v_{d}-u_{d} \widehat{f}_{r}\right] .
$$

where $k_{2}>0, \eta_{2}>0 . \hat{f}_{r}$ is the approximation of $f_{r}$ :

$$
\begin{aligned}
& \widehat{f}_{r}=\frac{1}{u_{d}} D^{1-\beta}\left(\widehat{W}_{r}^{T} \varphi\right), \\
& f_{r}=\frac{1}{u_{d}} D^{1-\beta}\left(W_{r}^{T} \varphi+\varepsilon_{2}\right),
\end{aligned}
$$

where $\varepsilon_{2}$ is the approximation error.

Subtracting (70) from (71),

$$
\begin{aligned}
f_{r}-\widehat{f}_{r} & =\frac{1}{u_{d}} D^{1-\beta}\left(W_{r}^{T} \phi+\varepsilon_{2}-\widehat{W}_{r}^{T} \phi\right) \\
& =\frac{1}{u_{d}} D^{1-\beta}\left[\left(W_{r}^{T}-\widehat{W}_{r}^{T}\right) \phi+\varepsilon_{2}\right] .
\end{aligned}
$$

Denote

$$
\widetilde{W}_{r}=W_{r}-\widehat{W}_{r}
$$

(72) can be written as

$$
f_{r}-\widehat{f}_{r}=\frac{1}{u_{d}} D^{1-\beta}\left(\tilde{W}_{r} \phi+\varepsilon_{2}\right) .
$$

The adaptive control rate is constructed:

$$
\dot{\hat{W}}_{r}=\frac{1}{\lambda_{2}} s_{2} \phi
$$

\subsection{Stability Analysis}

Theorem 2. With fractional-order sliding mode controllers (52) and (69) and adaptive control laws (58) and (75), the system converges in an asymptotically stable way.

Proof. Define the Lyapunov function:

$$
V=\frac{1}{2} s_{1}^{2}+\frac{1}{2} \lambda_{1} \tilde{W}_{u}^{T} \tilde{W}_{u}+\frac{1}{2} s_{2}^{2}+\frac{1}{2} \lambda_{2} \tilde{W}_{r}^{T} \tilde{W}_{r}
$$

The derivative of (76) can obtain

$$
\dot{V}=s_{1} \dot{s}_{1}+\lambda_{1} \widetilde{W}_{u}^{T} \dot{\widetilde{W}}_{u}+s_{2} \dot{s}_{2}+\lambda_{2} \widetilde{W}_{r}^{T} \dot{\widetilde{W}}_{r} .
$$

Substituting (31) into (30), one can obtain

$$
\begin{aligned}
\dot{s}_{1}(t) & =f_{u}+\dot{u}_{d}-c_{1} D^{1-\alpha} \bar{u}-\eta_{1} \operatorname{sgn}_{1} \\
& -\widehat{f}_{u}-\dot{u}_{d}+c_{1} D^{1-\alpha} \bar{u}=f_{u}-\widehat{f}_{u}-\eta_{1} \operatorname{sgn} s_{1} .
\end{aligned}
$$

Substituting (36) into (78), one can obtain

$$
\dot{s}_{1}(t)=\tilde{W}_{u}^{T} \varphi+\varepsilon_{1}-\eta_{1} \operatorname{sgn} s_{1} .
$$

Substituting (79) into (77), one can obtain 


$$
\begin{aligned}
\dot{V} & =s_{1}\left(\tilde{W}_{u}^{T} h+\varepsilon_{1}-\eta_{1} \text { sgn } s_{1}\right)+\lambda_{1} \tilde{W}_{u}^{T} \dot{\tilde{W}}_{u}+s_{2} \dot{s}_{2}+\lambda_{2} \tilde{W}_{r}^{T} \dot{\tilde{W}}_{r} \\
& =\varepsilon_{1} s_{1}-\eta_{1}\left|s_{1}\right|+\tilde{W}_{u}^{T}\left(s_{1} h+\lambda_{1} \dot{\tilde{W}}_{u}\right)+s_{2} \dot{s}_{2}+\lambda_{2} \tilde{W}_{r}^{T} \dot{\tilde{W}}_{r} .
\end{aligned}
$$

The derivative of (35) can obtain

$$
\dot{\widetilde{W}}_{u}=\dot{W}_{u}-\dot{\hat{W}}_{u}=-\dot{\widehat{W}}_{u} .
$$

Substituting (37) into (81), one can obtain

$$
\dot{\tilde{W}}_{u}=-\frac{1}{\lambda_{1}} s_{1} h
$$

Substituting (82) into (80), one can obtain

$$
\dot{V}=\varepsilon_{1} s_{1}-\eta_{1}\left|s_{1}\right|+s_{2} \dot{s}_{2}+\lambda_{2} \tilde{W}_{r}^{T} \dot{\tilde{W}}_{r} .
$$

Substituting (47) into (40), one can obtain

$$
\dot{s}_{2}(t)=c_{2} \dot{\bar{v}}+D^{\beta-1}\left[\ddot{v}-\left(\stackrel{\dot{t}}{y}_{d} \cos \psi-\sin \psi \stackrel{\dot{t}}{x}_{d}+\eta_{2} \ddot{\bar{x}} \sin \psi-\eta_{2} \ddot{\bar{y}} \cos \psi+r^{2} v_{d}-2 r \dot{u}_{d}-\dot{r} u_{d}\right)\right] .
$$

Substituting (12) into (84), one can obtain

$$
\begin{aligned}
\dot{s}_{2}(t) & =c_{2} \dot{\bar{v}}+D^{\beta-1}\left[\ddot{v}-\left(\ddot{y}_{d} \cos \psi-\sin \psi \ddot{x}_{d}+\eta_{2} \ddot{\bar{x}} \sin \psi-\eta_{2} \ddot{\bar{y}} \cos \psi+r^{2} v_{d}-2 r \dot{u}_{d}-f_{r} u_{d}-\frac{1}{m_{r}} \tau_{r} u_{d}\right)\right] \\
& =c_{2} \dot{\bar{v}}+D^{\beta-1} \ddot{v}-D^{\beta-1}\left(\ddot{y}_{d} \cos \psi-\sin \psi \ddot{x}_{d}+\eta_{2} \ddot{\bar{x}} \sin \psi-\eta_{2} \ddot{\bar{y}} \cos \psi+r^{2} v_{d}-2 r \dot{u}_{d}-f_{r} u_{d}-\frac{1}{m_{r}} \tau_{r} u_{d}\right) .
\end{aligned}
$$

Substituting (69) into (85), one can obtain

$$
\begin{aligned}
\dot{s}_{2}(t)= & c_{2} \dot{\bar{v}}+D^{\beta-1} \ddot{v}-D^{\beta-1}\left(\ddot{y}_{d} \cos \psi-\sin \psi \ddot{x}_{d}+\eta_{2} \ddot{\bar{x}} \sin \psi-\eta_{2} \ddot{\bar{y}} \cos \psi+r^{2} v_{d}-2 r \dot{u}_{d}-f_{r} u_{d}\right) \\
& +D^{\beta-1}\left[-\ddot{v}-D^{1-\beta}\left(k_{2} \operatorname{sgn} s_{2}+\eta_{2} s_{2}+c_{2} \dot{\bar{v}}\right)+\ddot{y}_{d} \cos \psi-\sin \psi \ddot{x}_{d}+\eta_{2} \ddot{\bar{x}} \sin \psi-\eta_{2} \ddot{\bar{y}} \cos \psi-2 r \dot{u}_{d}+r^{2} v_{d}-u_{d} \hat{f}_{r}\right] \\
= & -k_{2} \operatorname{sgn} s_{2}-\eta_{2} s_{2}+D^{\beta-1}\left(f_{r} u_{d}-u_{d} \widehat{f}_{r}\right) .
\end{aligned}
$$

Substituting (86) into (83), one can obtain

$$
\begin{aligned}
\dot{V} & =\varepsilon_{1} s_{1}-\eta_{1}\left|s_{1}\right|+s_{2}\left(-k_{2} \operatorname{sgns}_{2}-\eta_{2} s_{2}\right)+s_{2} D^{\beta-1}\left(f_{r} u_{d}-u_{d} \widehat{f}_{r}\right)+\lambda_{2} \widetilde{W}_{r}^{T} \dot{\tilde{W}}_{r} \\
& =\varepsilon_{1} s_{1}-\eta_{1}\left|s_{1}\right|-k_{2}\left|s_{2}\right|-\eta_{2} s_{2}^{2}+s_{2} D^{\beta-1}\left(f_{r} u_{d}-u_{d} \widehat{f}_{r}\right)+\lambda_{2} \widetilde{W}_{r}^{T} \dot{\tilde{W}}_{r} .
\end{aligned}
$$

Substituting (84) into (87), one can obtain

$$
\begin{aligned}
\dot{V} & =\varepsilon_{1} s_{1}-\eta_{1}\left|s_{1}\right|-k_{2}\left|s_{2}\right|-\eta_{2} s_{2}^{2}+s_{2} D^{\beta-1}\left[\frac{1}{u_{d}} D^{1-\beta}\left(\tilde{W}_{r} h+\varepsilon_{2}\right) u_{d}\right]+\lambda_{2} \tilde{W}_{r}^{T} \dot{\tilde{W}}_{r} \\
& =\varepsilon_{1} s_{1}-\eta_{1}\left|s_{1}\right|-k_{2}\left|s_{2}\right|-\eta_{2} s_{2}^{2}+s_{2}\left(\tilde{W}_{r} h+\varepsilon_{2}\right)+\lambda_{2} \tilde{W}_{r}^{T} \dot{\tilde{W}}_{r} .
\end{aligned}
$$


TABLE 2: Sea weather condition data.

\begin{tabular}{|c|c|c|c|c|c|c|}
\hline $\begin{array}{l}\text { Time of } \\
\text { observation }\end{array}$ & $\begin{array}{l}\text { Significant wave height } \\
(\mathrm{m})\end{array}$ & $\begin{array}{l}\text { Maximum wave height } \\
(\mathrm{m})\end{array}$ & $\begin{array}{c}\text { Wind speed } \\
(\mathrm{m} / \mathrm{s})\end{array}$ & $\begin{array}{l}\text { Maximum wind speed } \\
(\mathrm{m} / \mathrm{s})\end{array}$ & $\begin{array}{c}\text { Wind } \\
\text { direction }\end{array}$ & $\begin{array}{l}\text { Wind } \\
\text { force }\end{array}$ \\
\hline $07.50 \mathrm{PM}$ & 1.40 & 2.20 & 9.9 & 12.6 & $\begin{array}{c}\text { North- } \\
\text { northeast }\end{array}$ & 5 \\
\hline 08.00 PM & 1.60 & 2.40 & 10.40 & 12.60 & North & 5.00 \\
\hline $08.10 \mathrm{PM}$ & 1.60 & 2.40 & 10.80 & 14.10 & $\begin{array}{l}\text { North- } \\
\text { northeast }\end{array}$ & 6.00 \\
\hline 08.20 PM & 1.60 & 2.40 & 11.50 & 13.80 & $\begin{array}{c}\text { North- } \\
\text { northeast }\end{array}$ & 6.00 \\
\hline 08.30 PM & 1.70 & 2.20 & 10.60 & 13.70 & $\begin{array}{c}\text { North- } \\
\text { northeast }\end{array}$ & 5.00 \\
\hline 08.40 PM & 1.70 & 2.20 & 10.60 & 13.30 & North & 5.00 \\
\hline
\end{tabular}

The derivative of (83) can obtain

$$
\dot{\widetilde{W}}_{r}=\dot{W}_{r}-\dot{\widehat{W}}_{r}=-\dot{\widehat{W}}_{r} .
$$

Substituting (75) into (89), one can obtain

$$
\dot{\tilde{W}}_{r}=-\frac{1}{\lambda_{2}} s_{2} \varphi .
$$

Substituting (86) into (84), one can obtain
TABle 3: Tidal height on Dec. 30, 2019.

\begin{tabular}{lc}
\hline Tide time & Tidal height \\
\hline 12.00 AM & 447 \\
01.00 AM & 516 \\
02.00 AM & 549 \\
02.04 AM & 549 \\
03.00 AM & 524 \\
04.00 AM & 434 \\
05.00 AM & 319 \\
\hline
\end{tabular}

$$
\begin{aligned}
\dot{V} & =\varepsilon_{1} s_{1}-\eta_{1}\left|s_{1}\right|-k_{2}\left|s_{2}\right|-\eta_{2} s_{2}^{2}+s_{2}\left(\tilde{W}_{r} h+\varepsilon_{2}\right)-\tilde{W}_{r}^{T} s_{2} \varphi \\
& =\varepsilon_{1} s_{1}-\eta_{1}\left|s_{1}\right|-k_{2}\left|s_{2}\right|-\eta_{2} s_{2}^{2}+\varepsilon_{2} s_{2} .
\end{aligned}
$$

The following conditions are established:

$$
\begin{aligned}
& \left|\varepsilon_{1}\right| \leq \eta_{1}, \\
& \left|\varepsilon_{2}\right| \leq k_{2} .
\end{aligned}
$$

We have

$$
\dot{V} \leq 0
$$

The system is asymptotically stable, given that the stability of the presented method cannot be sustained due to the dynamic memory of the fractional operators.

\section{Simulation Studies}

A container ship [19] is used for testing. The length of the ship is $175 \mathrm{~m}$. Table 2 lists sea weather condition data.

Table 3 lists the tidal height on Dec. 30, 2019.

Table 4 lists the ship collision avoidance parameters between two ships.

4.1. Results. Figure 4 shows the speed response curve of ship 1.

Figure 5 shows the position and attitude response curve of ship 1
Figure 6 shows the roll response curve of ship 1 . Figure 7 shows the input rudder angle of ship 1. Figure 8 shows the speed response curve of ship 2 .

Figure 9 shows the position and attitude response curve of ship 1.

Figure 10 shows the roll response curve of ship 2 .

Figure 11 shows the input rudder angle of ship 2 .

Figure 12 shows the resultant positions of ships 1 and 2 to avoid collisions.

Figures 4-12 show that FRNN-AFSMC can realize the collision avoidance between two ships.

4.2. Performance Comparison. Table 4 shows the performance comparison of different algorithms, including SMC, ASMC with RNN (RNN-ASMC), and FRNN-AFSMC.

Table 5 shows that FRNN-AFSMC has less overshoot and a shorter system regulation time with a wider parameter selection range.

Figure 13 shows the yawing response curve of the closedloop control system, where the horizontal axis represents the time in seconds and the longitudinal axis represents the yaw angle in unit of rad.

Figure 13 shows that, compared with basic sliding mode control algorithm, the overshoot of FRNN-AFOSMC is smaller and the regulation time of the system is shorter. 
TABle 4: Ship collision avoidance parameters.

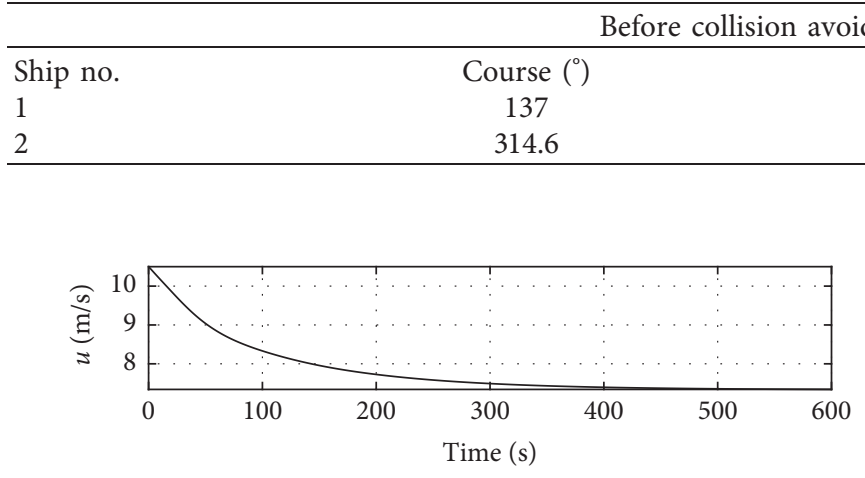

(a)

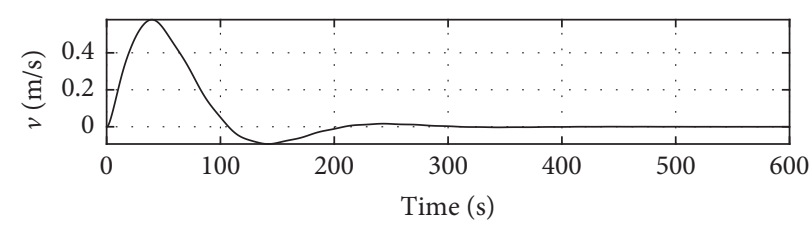

(b)

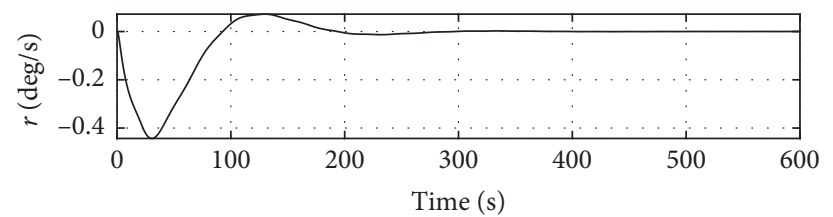

(c)

FIgURE 4: Speed response curve of ship 1.

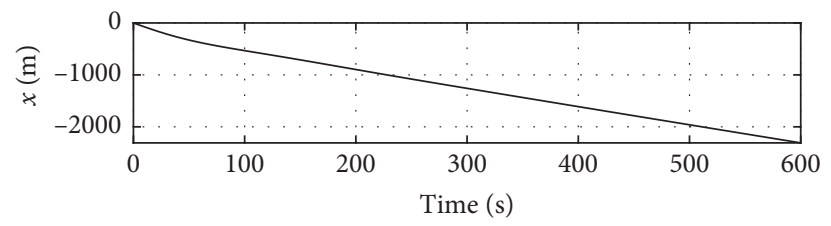

(a)

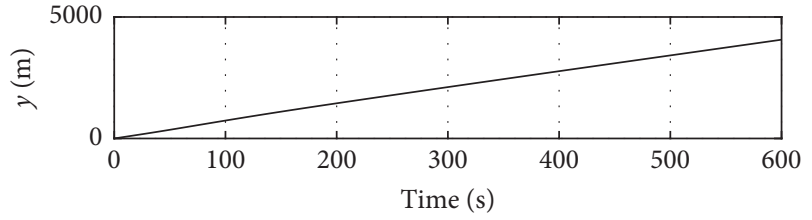

(b)

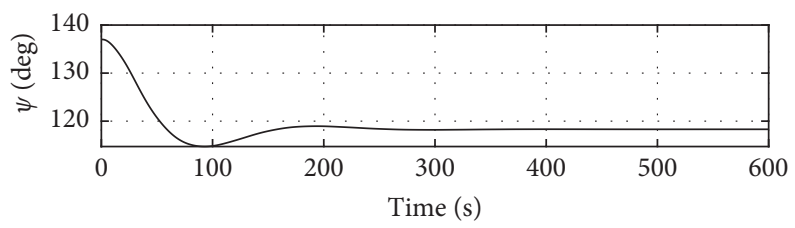

(c)

Figure 5: Position and attitude response of ship 1.

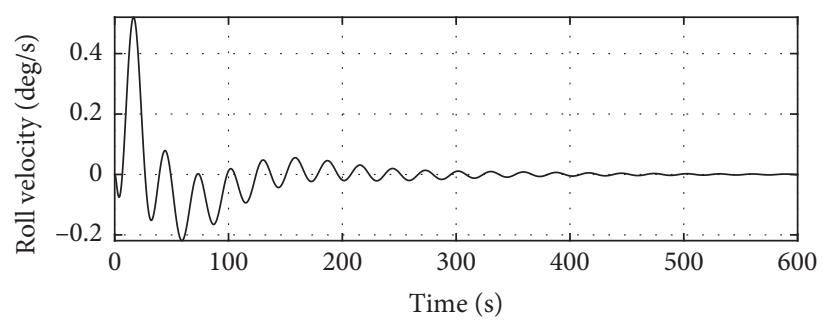

(a)

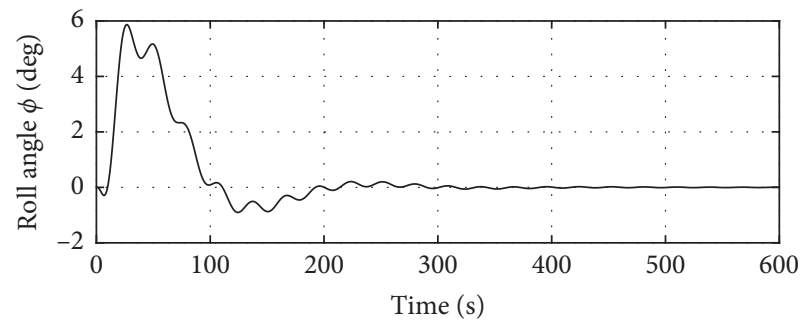

(b)

Figure 6: Roll response curve of ship 1. 


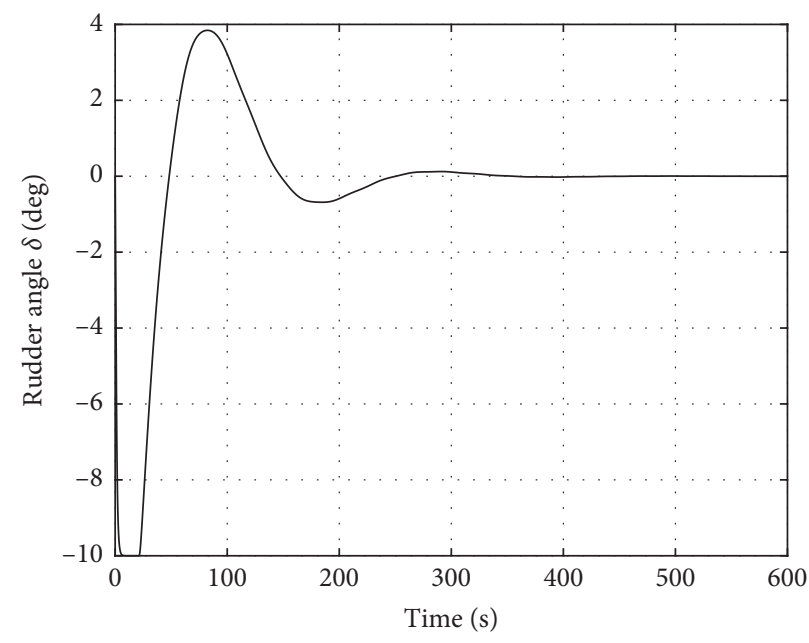

Figure 7: Input rudder angle curve of ship 1.

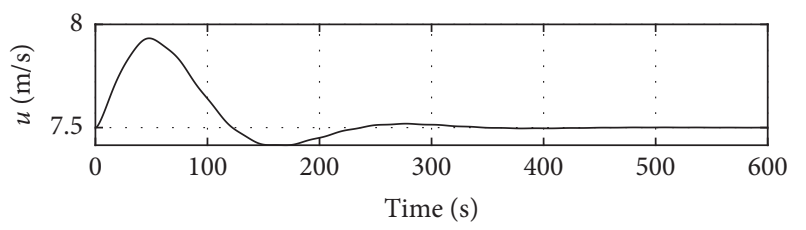

(a)

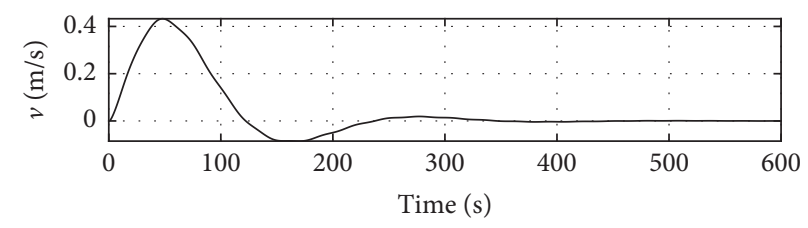

(b)

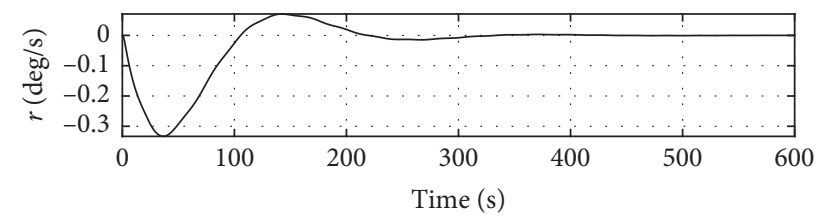

(c)

FIGURE 8: Speed response curve of ship 2.

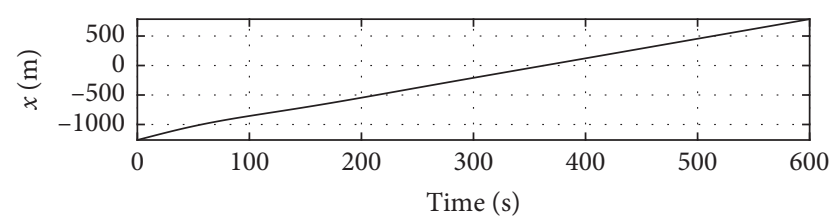

(a)

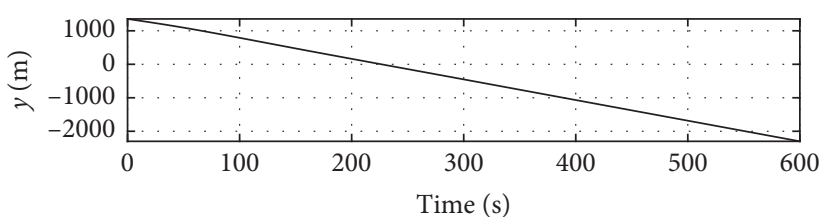

(b)

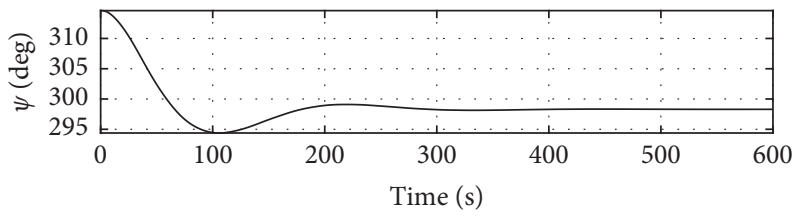

(c)

Figure 9: Position and attitude response curve of ship 2. 


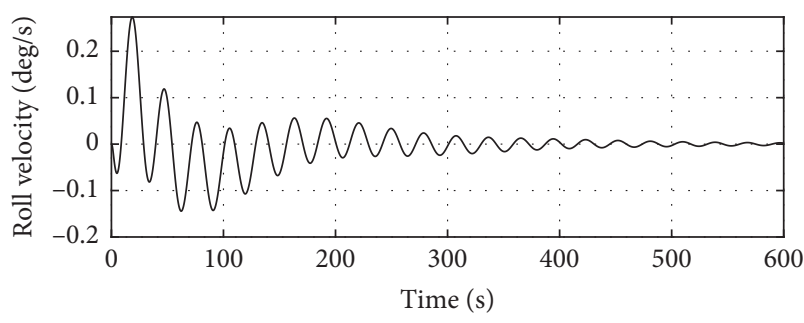

(a)

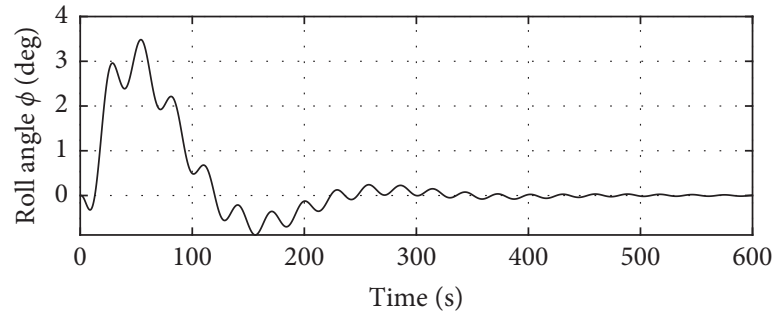

(b)

Figure 10: Roll response curve of ship 2.

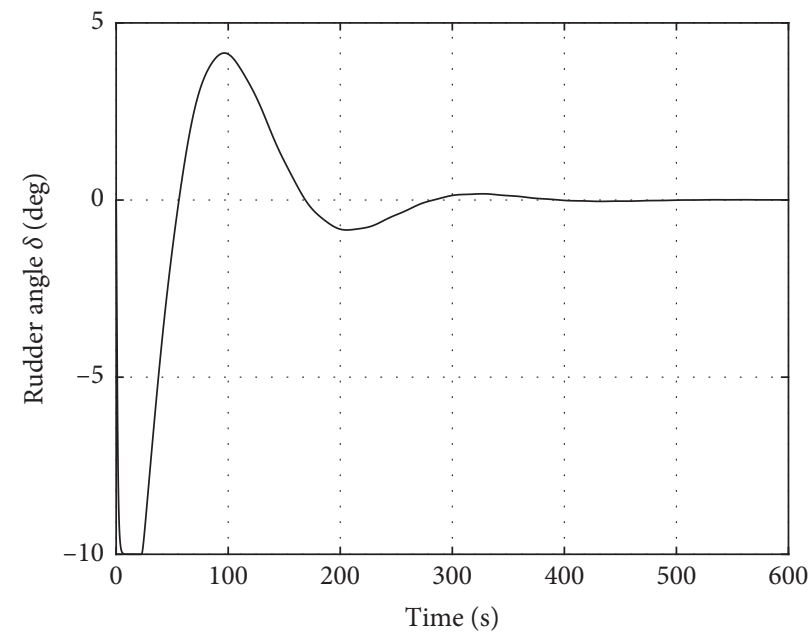

Figure 11: Input rudder angle curve of ship 2.

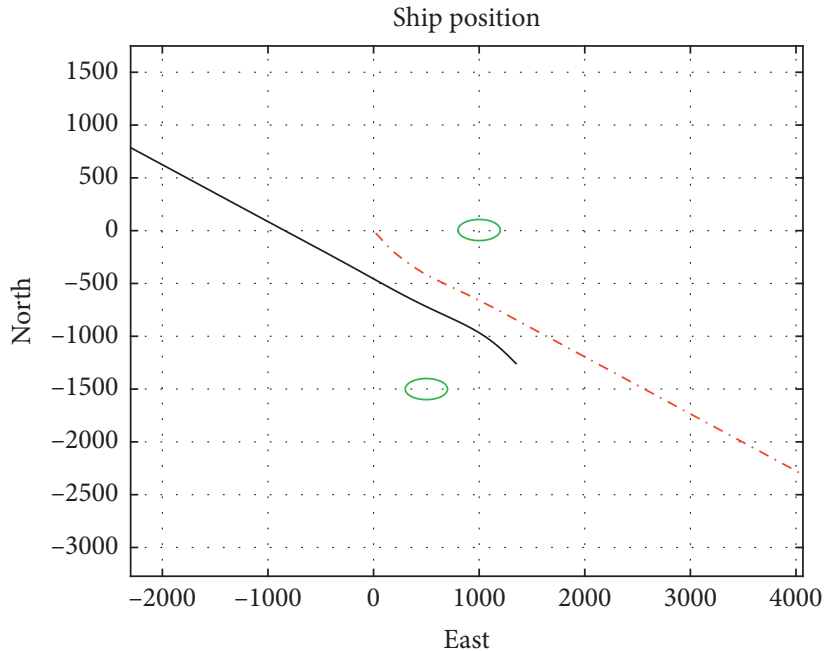

-..- Ship 1

- Ship 2

Figure 12: Position of two ships.

TABLE 5: Comparison of the control performances of different algorithms.

\begin{tabular}{lccc}
\hline & SMC & RNN-ASMC & FRNN-AFOSMC \\
\hline Overshoot & $19 \%$ & $13 \%$ & $9 \%$ \\
Adjustment time (s) & 192 & 176 & 156 \\
\hline
\end{tabular}




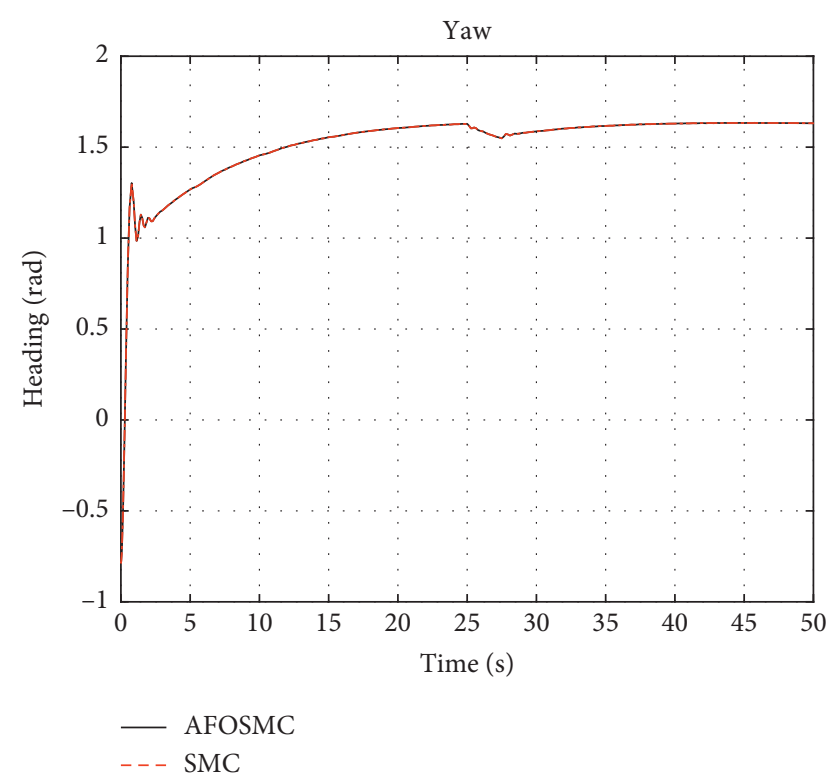

FIgURe 13: Performance comparison of different algorithms.

\section{Conclusions}

In this paper, FRNN-AFSMC is proposed and shown to converge for collision avoidance in bridge areas. The uncertainties are estimated by FRNN. The influence of fractional order on the algorithm performance is analyzed. Multiple ships dynamic collision avoidance is simulated and verified. The results show the performance comparison of different algorithms, including SMC, ASMC with RNN (RNN-ASMC), and FRNN-AFSMC. The accuracy of the control algorithm will be improved in future research.

\section{Data Availability}

The data used are included within the manuscript.

\section{Conflicts of Interest}

The authors declare that they have no conflicts of interest.

\section{Acknowledgments}

This work was supported by Xiamen Social Science Research Project from Xiamen Federation of Social Sciences and Xiamen Academy of Social Sciences (XMSK2021C04).

\section{References}

[1] B. Wu, T. L. Yip, X. P. Yan, and C. G. Soares, "Fuzzy logic based approach for ship-bridge collision alert system," Ocean Engineering, vol. 187, Article ID 106152, 2019.

[2] L. Li, Z.-M. Yuan, C. Ji, M.-X. Li, and Y. Gao, "Investigation on the unsteady hydrodynamic loads of ship passing by bridge piers by a 3-D boundary element method," Engineering Analysis with Boundary Elements, vol. 94, pp. 122-133, 2018.

[3] Y. Y. Sha, J. Amdahl, and K. Liu, "Design of steel bridge girders against ship forecastle collisions," Engineering Structures, vol. 196, Article ID 109277, 2019.
[4] P. T. Pedersen, J. Chen, and L. Zhu, "Design of bridges against ship collisions," Marine Structures, vol. 74, Article ID 102810, 2020.

[5] H. Fang, Y. Mao, W. Liu, L. Zhu, and B. Zhang, "Manufacturing and evaluation of Large-scale Composite Bumper System for bridge pier protection against ship collision," Composite Structures, vol. 158, pp. 187-198, 2016.

[6] L. Zhu, W. Liu, H. Fang, J. Chen, Y. Zhuang, and J. Han, "Design and simulation of innovative foam-filled Lattice Composite Bumper System for bridge protection in ship collisions," Composites Part B: Engineering, vol. 157, pp. 2435, 2019.

[7] J. Fei and H. Wang, "Recurrent neural network fractionalorder sliding mode control of dynamic systems," Journal of the Franklin Institute, vol. 357, no. 8, pp. 4574-4591, 2020.

[8] L. Y. Xiong, P. H. Li, M. L. Ma, Z. Q. Wang, and J. Wang, "Output power quality enhancement of PMSG with fractional order sliding mode control," Electrical Power and Energy Systems, vol. 115, Article ID 105402, 2020.

[9] A. Sharafian, A. Sharifi, and W. Zhang, "Fractional sliding mode based on RBF neural network observer: application to HIV infection mathematical model," Computers \& Mathematics with Applications, vol. 79, no. 11, pp. 3179-3188, 2020.

[10] B. Yang, J. B. Wang, Y. Y Sang et al., "Applications of supercapacitor energy storage systems in microgrid with distributed generators via passive fractional-order slidingmode control," Energy, vol. 187, Article ID 115905, 2019.

[11] S. A. Moezi, E. Zakeri, and M. Eghtesad, "Optimal adaptive interval type-2 fuzzy fractional-order backstepping sliding mode control method for some classes of nonlinear systems," ISA Transactions, vol. 93, pp. 23-39, 2019.

[12] H.-P. Ren, X. Wang, J.-T. Fan, and O. Kaynak, "Fractional order sliding mode control of a pneumatic position servo system," Journal of the Franklin Institute, vol. 356, no. 12, pp. 6160-6174, 2019.

[13] J. L. Elman, "Finding structure in time," Cognitive Science, vol. 14, no. 2, pp. 179-211, 1990.

[14] A. Sharafian and R. Ghasemi, "Fractional neural observer design for a class of nonlinear fractional chaotic systems," Neural Computing and Applications, vol. 31, no. 4, pp. 12011213, 2019.

[15] A. Sharafian, A. Sharifi, and W. Zhang, "Different types of sliding mode controller for nonlinear fractional multi-Agent system," Chaos, Solitons \& Fractals, vol. 131, Article ID 109481, 2020.

[16] I. S. Jesus and R. S. Barbosa, "Design of fuzzy fractional PD plus I controllers tuned by a genetic algorithm," in Proceedings of the 39th Annual Conference of the IEEE Industrial Electronics society, IEEE, Vienna, Austria, November 2013.

[17] R. Abiyev, "Design of fractional order controllers using genetic algorithms," British Journal of Mathematics \& Computer Science, vol. 4, no. 18, pp. 2594-2602, 2014.

[18] H. Xue and T. Chai, "Vessel track prediction based on fractional gradient recurrent neural network with maneuvering behavior identification," Scientific Programming, vol. 2021, Article ID 5526082, 11 pages, 2021

[19] T. I. Fossen, Marine Control System: Guidance, Navigation and Control of Ships, Rigs and Underwater Vehicles, Springer, Berlin, Germany, 2002. 